\title{
Long-Term Results after Suprapubic ARC Procedure for the Treatment of Stress Urinary Incontinence in Women: A Retrospective Data Analysis
}

\author{
Samra Jasarevic ${ }^{a}$ Doroteja Jankovic ${ }^{a}$ Georg C. Hutterer ${ }^{a}$ Regina Riedl ${ }^{b}$ \\ Georg P. Pichler ${ }^{a}$ Karl Pummer ${ }^{a}$ Günter Primus ${ }^{a}$

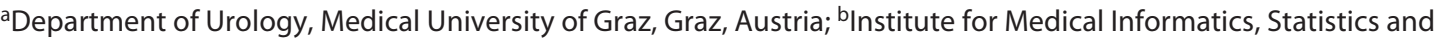 \\ Documentation, Medical University of Graz, Graz, Austria
}

\section{Keywords \\ Long-term outcome - Mid-urethral sling · Suprapubic arc · \\ Stress urinary incontinence}

\begin{abstract}
Introduction: The aim of this study was to evaluate longterm safety and efficacy of the suprapubic arc (SPARC) procedure for the surgical treatment of stress urinary incontinence (SUI). Materials and Methods: 139 female patients treated by SPARC were included in this retrospective analysis, whereby 126 patients were available for follow-up after 1 year, 70 after 6 years, and 41 after 9 years. The cough test, pad test, uroflowmetry, and post-void residual volume measurements were performed. Severity of bother (visual analogous scale [VAS] 0-10), continence, and the satisfaction rate were assessed. Objective cure was defined as a negative cough test and pad weight $\leq 1 \mathrm{~g}$, subjective cure as no urine loss during daily activities and no usage of pads. The VAS, pad weight, number of pads per day, and maximal flow rate were compared preoperatively and postoperatively. $\boldsymbol{R e}$ sults: Objective cure rates at 1, 6, and 9 years were 78.6, 71.4, and $70.7 \%$ and subjective cure rates were $72.2,55.7$, and $65.8 \%$, respectively. The VAS, pad weight, number of pads,
\end{abstract}

karger@karger.com www.karger.com/uin

Karger $\stackrel{\text { ' }}{5}$
(C) 2021 The Author(s)

Published by S. Karger AG, Basel

This is an Open Access article licensed under the Creative Common Attribution-NonCommercial-4.0 International License (CC BY-NC) (http://www.karger.com/Services/OpenAccessLicense), applicable to the online version of the article only. Usage and distribution for commercial purposes requires written permission. and maximal flow rate decreased significantly. Study limitations include a relatively small sample size and the retrospective fashion of the analysis. Conclusions: In the long-term context, SPARC showed to represent an efficient and safe procedure for treatment of female SUI.

(c) 2021 The Author(s)

Published by S. Karger AG, Basel

\section{Introduction}

Stress urinary incontinence (SUI), defined as an involuntary leakage of urine taking place synchronously with physical effort, exertion, sneezing, coughing, or laughing [1], represents by far the most common form of incontinence in women. Moreover, with a prevalence of $12-46 \%$ in adult women, SUI constitutes a great economic burden for the affected individuals, as well as the healthcare-providing systems [2].

In general, conservative treatment modalities including pelvic floor muscle training and weight loss should be offered as first-line therapy, but in many cases, the achieved effects are not sufficient. In this context, a trend toward minimally invasive surgical techniques led to a 
renaissance of various methods of urethral bulking over the last years. Since its effectiveness is lower than other more invasive operative methods, urethral bulking still represents a valid treatment option for elderly and fragile patients or patients who are regarded unfit for surgery for other reasons with mild to moderate incontinence $[3,4]$.

Mid-urethral slings have shown good efficacy in a large number of patients and thus now represent a mainstay in the surgical management of female SUI $[5,6]$. Among those, tension-free vaginal tapes (TVTs) have been the most frequently used methods since their introduction in the 1990s [7]. Numerous studies investigated the safety and efficacy of this surgical technique, providing good short- and long-term data $[8,9]$.

The suprapubic arc (SPARC) sling procedure (American Medical Systems, Minnetonka, MN, USA) was first introduced in 2001 and used successfully at our department from 2004 to 2018 . The production of SPARC slings by AMS was closed in 2016 due to nonmedical reasons.

SPARC and TVTs represent comparable surgical methods in terms of subjective cure/improvement and patients' satisfaction rates, with SPARC showing less voiding impairment, a higher likelihood of a positive stress test, and a higher rate of bladder and urethral injuries, with the latter possibly in part due to surgeons' limited experience with the SPARC procedure $[2,10]$. SPARC trocars are placed using a suprapubic approach via 2 small incisions above the pubic bone to the vaginal incision below the mid-urethra. In particular, the so-called "top-tobottom" approach might induce less vessel injuries, making SPARC a possibly safer procedure concerning bleeding complications than TVTs [11]. Until today, only few studies have reported short- (up to 1 year) and mediumterm (1-5 years) safety and efficacy data of the SPARC procedure [10-14]. Published long-term data in this context are scarce [15]. At the moment, long-term safety data of mid-urethral slings represent a paramount issue within the field of incontinence surgery, since efficacy and safety of these have been re-evaluated and even litigated or suspended in some countries [16]. The working group on the Urological Functional Diagnostics and Female Urology of the Academy of the German Society of Urology enunciated in their last statement unrestricted recommendation for the mid-urethral slings as there is a clear difference between these and mesh implants, with mid-urethral slings showing good efficacy and safety profile [17]. The aim of this retrospective study was to evaluate long-term safety and efficacy of the SPARC procedure for the treatment of female SUI at a tertiary academic center.

\section{Materials and Methods}

Electronic patient records from 139 female patients treated with the SPARC procedure for SUI between 2001 and 2014 at the Department of Urology of the Medical University of Graz were evaluated retrospectively. Complete data from 126 patients were available for follow-up evaluation after 1 year, complete data from 70 patients after 5 to 6 years, and from 41 patients after 7 to 10 years. All patients suffered from pure or mixed SUI, with the stress component being in the foreground. Primarily, all patients of the study cohort were treated insufficiently by pelvic floor exercise. In patients with mixed urinary incontinence, the urgency component was treated with antimuscarinics. If the urgency component was treated successfully, patients underwent the SPARC procedure subsequently. Women undergoing their first incontinence operation, as well as those who experienced an unsuccessful incontinence surgery previously, were included into this study.

Exclusion criteria were pure urgency incontinence, chronic urinary retention with incontinence, various neurological findings with a significant impact on the lower urinary tract function, continuous urinary incontinence, and pelvic organ prolapse $>2$ according to the Pelvic Organ Prolapse Quantification System. Preoperatively, all patients underwent cystoscopy and invasive urodynamic studies, performed with the urodynamic system Ellipse Andromeda, at the Department of Urology of the Medical University of Graz. The invasive urodynamic studies were repeated 1 year postoperatively.

A cough stress test with a bladder filling of at least $250 \mathrm{~mL}$, the pad weight test according to Hahn \& Fall, and uroflowmetry with post-void residual volume (PVR) measurements were performed preoperatively, as well as during the follow-up visits. The primary outcome was objective and subjective cure rates. Objective cure was defined as a pad weight $0-1 \mathrm{~g}$ and a negative cough stress test in standing position. Subjective cure was defined as no use of pads according to the micturition diary and no urine loss during daily activities. Secondary outcomes included patient-reported continence and satisfaction rates, de novo urgency symptoms, evaluation of lower urinary tract infections, and the assessment of the incontinence-related quality of life. A standardized questionnaire according to the Visual Analog Scale (VAS) from 0 (not bothersome) to 10 (most bothersome) was used to access the severity of bother regarding SUI. Patients' satisfaction with the outcome of the SPARC procedure was evaluated using patient interviews at the visits, with possible responses as "very satisfied," "satisfied," and "dissatisfied." In addition, patients had to describe themselves as being "continent," "minor incontinent," or "incontinent". Moreover, we asked the patients whether they would be willing to undergo this particular surgical procedure again and whether they would recommend it to a friend. Additionally, we analyzed the subgroups regarding previous pelvic and/or incontinence surgery.

\section{Statistical Analysis}

Continuous parameters are presented as median, minimum, and maximum and categorical data as frequency and percent. The Wilcoxon signed-rank test was used to evaluate changes in the VAS, pad test weight, number of pads per day, and maximum flow rate from baseline to follow-up (1, 6, and 9 years). Group comparisons were performed for the objective and subjective cure rates during the follow-up by using multivariable logistic regression 
Table 1. Overview of complications

\begin{tabular}{|c|c|c|c|}
\hline & \multicolumn{3}{|l|}{$N(\%)$} \\
\hline \multicolumn{4}{|l|}{ Intraoperative } \\
\hline Bladder perforation & $4 / 139$ (2.9) & & \\
\hline \multicolumn{4}{|l|}{ Early postoperative } \\
\hline Urinary retention & $3 / 139(2.2)$ & & \\
\hline PVR & $6 / 139(4.3)$ & & \\
\hline Tape gliding & $3 / 139(2.2)$ & & \\
\hline Hematoma & 0 & & \\
\hline Urinary infections & 0 & & \\
\hline Late postoperative & 1 year & 6 years & 9 years \\
\hline De novo urgency & $2 / 126(1.6)$ & $7 / 70(10.0)$ & $4 / 41(9.8)$ \\
\hline Recurrent urinary infections & 0 & $1 / 70(1.4)$ & $3 / 41(7.3)$ \\
\hline Sling exposure (vaginal) & 0 & $2 / 70(2.9)$ & 0 \\
\hline
\end{tabular}

PVR, post-void residual volume.

analysis adjusting for age, BMI, and partus. A $p$ value $<0.05$ is considered statistically significant. Statistical analysis was performed using IBM SPSS Statistics 26.0.

\section{Results}

In all cases, the SPARC procedure was performed under general (122/139, 87.8\%) or spinal anesthesia (17/139, $12.2 \%)$ by one dedicated and experienced urologic surgeon. Follow-up periods were 1 year, 5-6 (median 6 years), and 7-10 (median 9 years). Overall, patients' median age was $62(34-91)$ years, the median BMI was 27.8 $(17.8-42.0) \mathrm{kg} / \mathrm{m}^{2}$, and the median number of partus was $2(0-5)$ at the date of surgery. Thirteen of 139 (9.4\%) patients had mixed urinary incontinence. Forty-three of 139 (30.9\%) had recurrent urinary incontinence after previous incontinence surgery. In 79/139 (56.8\%) patients, pelvic surgeries were performed previously (for details see online suppl. Table 1; for all online suppl. material, see www.karger.com/doi/10.1159/000516941). The overview of complications is listed in Table 1 . Objective cure rates were 99/126 (78.6\%), 50/70 (71.4\%), and 29/41 $(70.7 \%)$ at the 1-, 6-, and 9-year follow-ups, respectively. Subjective cure rates were 91/126 (72.2\%), 39/70 (55.7\%), and $27 / 41(65.9 \%)$ at the 1-, 6-, and 9-year follow-ups, respectively.

At 1-year follow-up, 123/126 (97.6\%) described themselves as continent and $3 / 126(2.4 \%)$ as incontinent. $120 / 126(95.2 \%)$ were very satisfied with the surgical outcome, and 6/126 (4.8\%) were satisfied.

Long-Term Results after SPARC Procedure for the Treatment of Female SUI
Table 2. PVR $>0 \mathrm{~mL}$

\begin{tabular}{lll}
\hline & $N(\%)$ & Range, $\mathrm{mL}$ \\
\hline Preoperatively & $4 / 139(2.9)$ & $50-80$ \\
1 year & $5 / 126(4.0)$ & $40-150$ \\
6 years & $6 / 70(8.6)$ & $20-100$ \\
9 years & $4 / 41(9.6)$ & $50-280$ \\
\hline
\end{tabular}

PVR, post-void residual volume.

At 6-year follow-up, 47/70 (67.1\%) described themselves as continent, $20 / 70(28.6 \%)$ as mildly incontinent, and $3 / 70(4.3 \%)$ as incontinent. One of these incontinent patients had developed a band exposure and one had an insensitive detrusor with overflow incontinence caused by cerebral dysfunction. Thirty of 70 (42.9\%) were using pads during their daily activities; however, 14 of these 30 patients (46.7\%) were using only 1 security pad per day. Forty-seven of $70(67.1 \%)$ were very satisfied with the surgical outcome, 18/70 (25.7\%) were satisfied, and 5/70 (7.1\%) were dissatisfied.

At 9-year follow-up, 31/41 (75.6\%) described themselves as continent and 10/41 (24.4\%) as mildly incontinent. Fourteen of 41 (34.1\%) were using pads; however, 6 of these 14 patients $(42.9 \%)$ were using only 1 security pad per day. Thirty-one of 41 (75.6\%) were very satisfied with the surgical outcome, and 10/41 (24.4\%) were satisfied.

During all follow-up periods, all patients reported that they would have recommended the SPARC procedure to a friend. With the exception of 1 patient at 6 years of follow-up, all patients reported that they would have performed the SPARC procedure again. The VAS decreased from $8(2-10)$ to $0(0-10)$, from $8(2-10)$ to $1(0-10)$, and from 8 (4-10) to $0(0-8)$ at 1-, 6-, and 9-year follow-ups, respectively $(p<0.001)$ (shown in Fig. 1 ).

The median pad weight decreased from $16 \mathrm{~g}(0-743)$ to $0 \mathrm{~g}(0-85)$, from $12 \mathrm{~g}(1-743)$ to $0 \mathrm{~g}(0-27)$, and from $15 \mathrm{~g}(1-354)$ to $0 \mathrm{~g}(0-7)$ at the 1-, 6-, and 9-year followups, respectively $(p<0.001)$ (shown in Fig. 2). The median number of pads per day decreased from 4 (1-20) to $0(0-10)$, from $4(1-20)$ to $0(0-5)$, and from $4(1-12)$ to $0(0-5)$ at the 1-, 6-, and 9-year follow-ups, respectively $(p<0.001)$ (shown in Fig. 3).

The maximum flow rate decreased from $37.5 \mathrm{~mL} / \mathrm{s}$ (11-85.6) to $34.7 \mathrm{~mL} / \mathrm{s}(8-70), 25.0 \mathrm{~mL} / \mathrm{s}(3.4-60.9)$, and $20.3 \mathrm{~mL} / \mathrm{s}(2.4-70.1)$ at the 1-, 6-, and 9-year follow-ups, respectively $(p<0.001)$ (shown in Fig. 4). Finally, we did not observe any subjective impairment due to the reduc- 


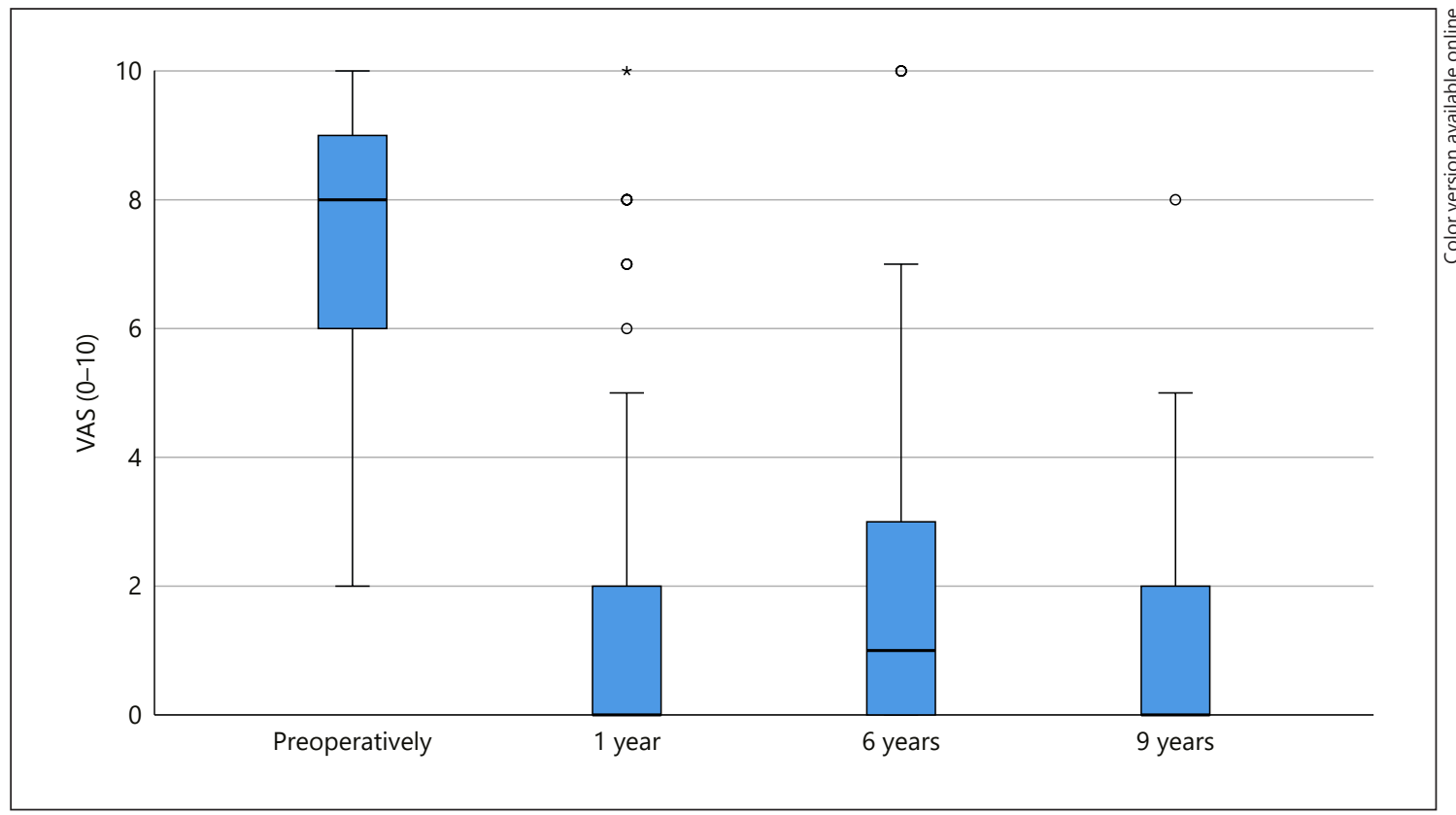

Fig. 1. Comparison of severity of bother regarding SUI according to VAS $0-10$ preoperatively $(n=139)$, at 1 $(n=126), 6-(n=70)$, and 9-year $(n=41)$ follow-ups. $p$ values were calculated for each follow-up period comparing it to the particular baseline. All $p<0.001$. SUI, stress urinary incontinence; VAS, visual analogous scale.

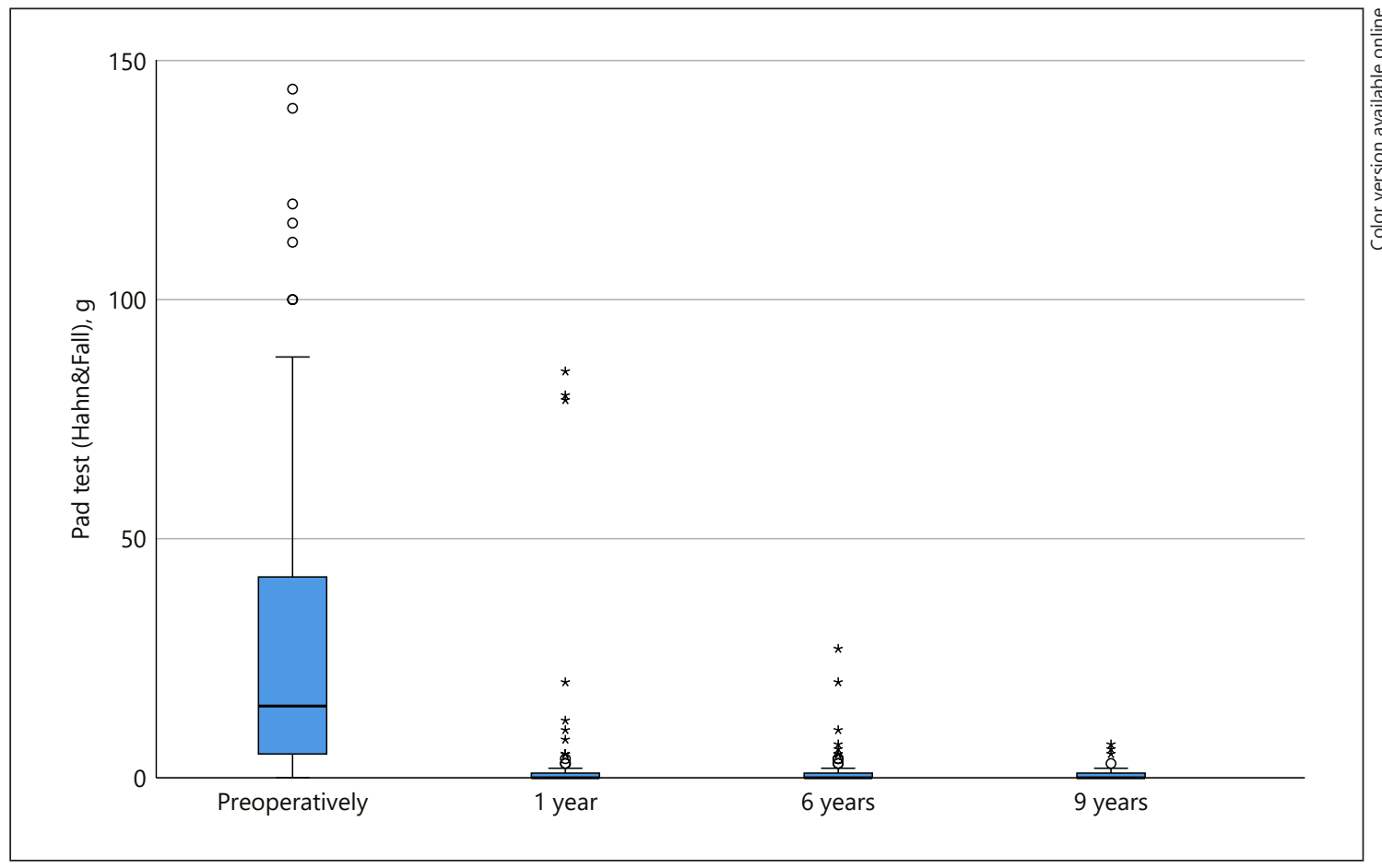

Fig. 2. Comparison of pad weight in gram according to Hahn \& Fall preoperatively $\left(n=133^{*}\right)$, at $1-(n=126)$, 6- $(n=70)$, and 9-year $(n=41)$ follow-ups. *For the better overview, 6 outliers were removed from the figure. $p$ values were calculated for each follow-up period comparing it to the particular baseline. All $p<0.001$. 


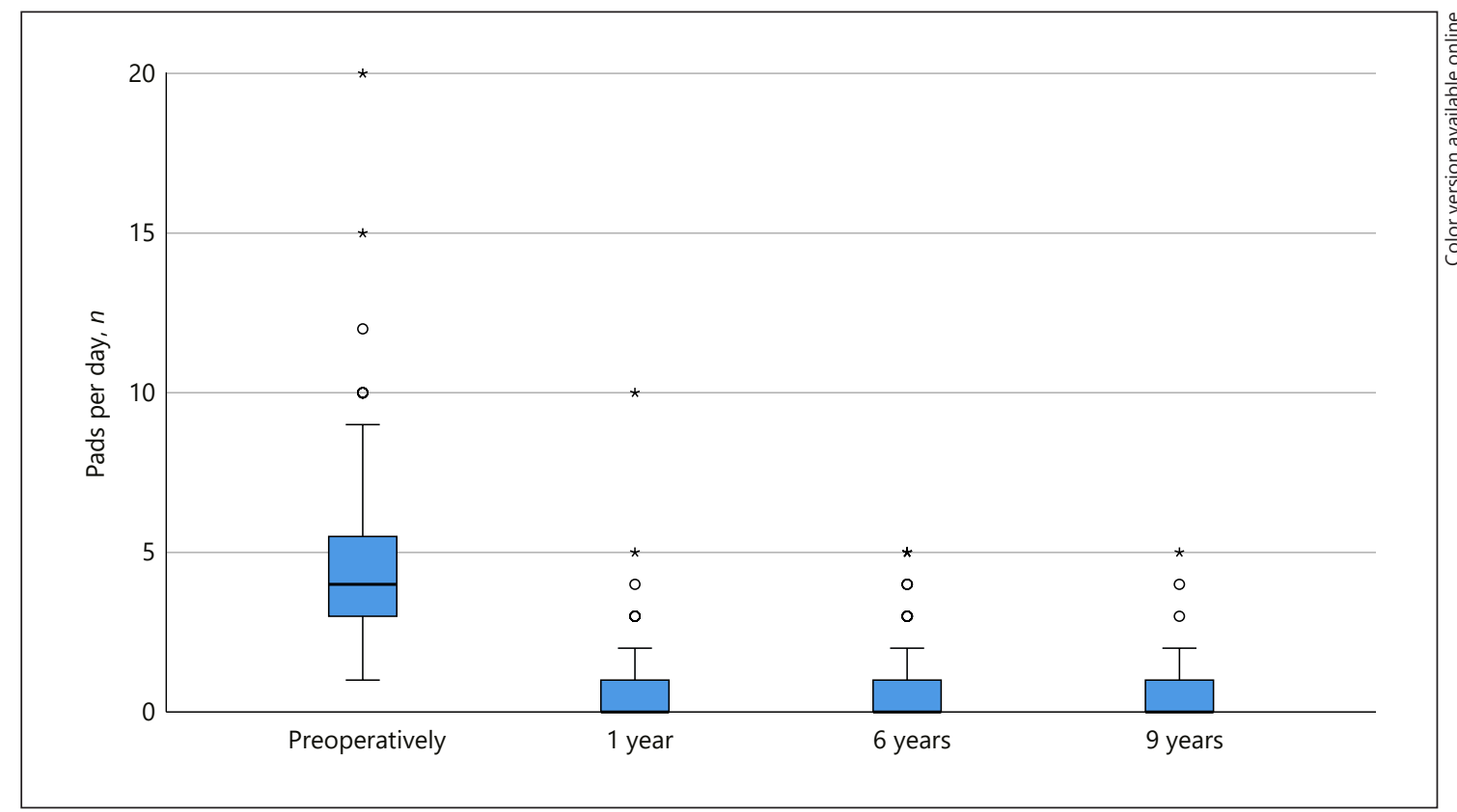

Fig. 3. Comparison of number of pads used per day preoperatively $(n=139)$, at 1 - $(n=126)$, 6- $(n=70)$, and 9 -year $(n=41)$ follow-ups. $p$ values were calculated for each follow-up period comparing it to the particular baseline. All $p<0.001$.

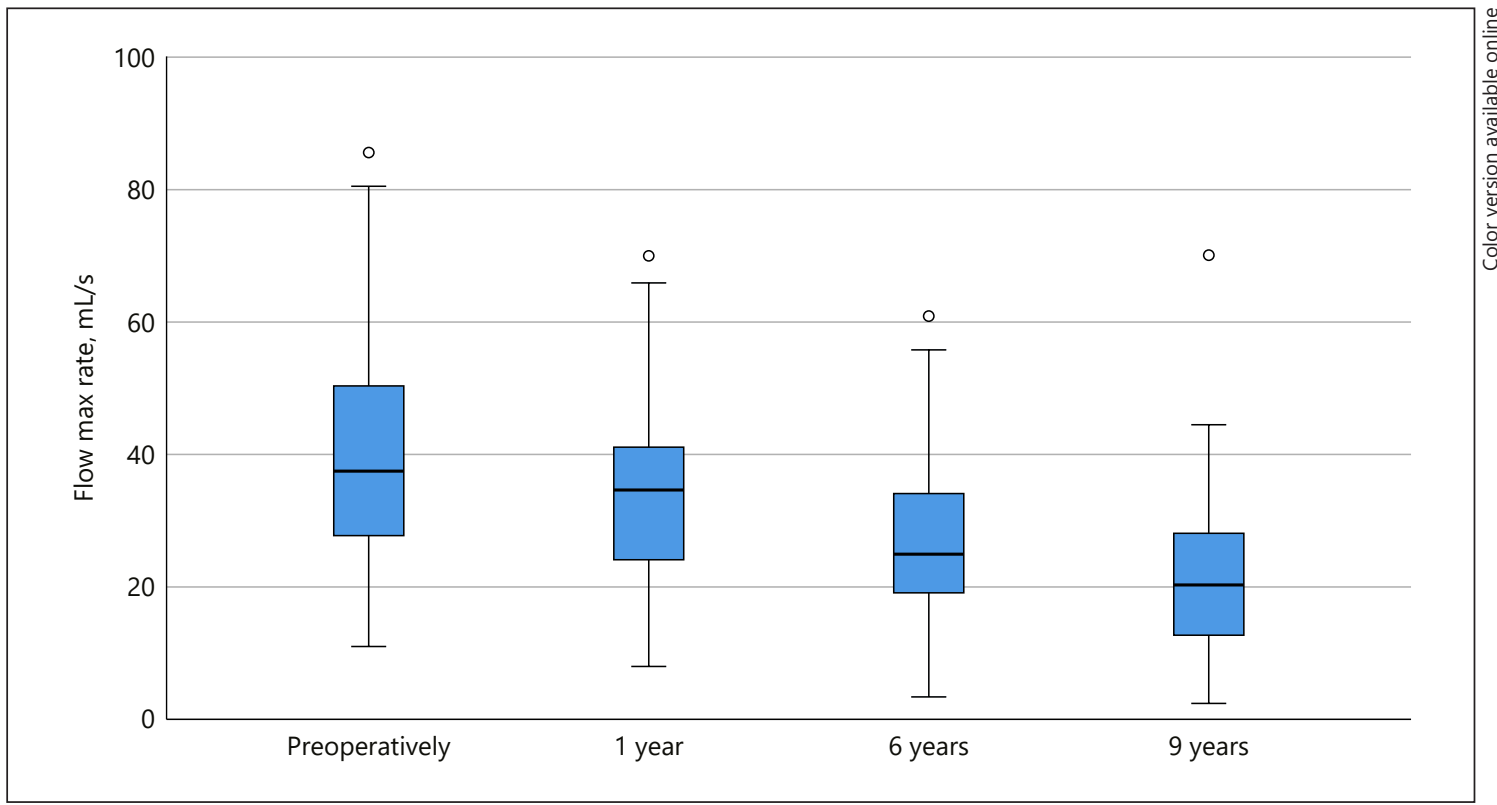

Fig. 4. Comparison of the maximal flow rate preoperatively $(n=139)$, at $1-(n=126)$, 6- $(n=70)$, and 9-year $(n=41)$ follow-ups. $p$ values were calculated for each follow-up period comparing it to the particular baseline. All $p<0.001$. 
Table 3. Analysis of subgroups regarding previous pelvic and/or incontinence surgery

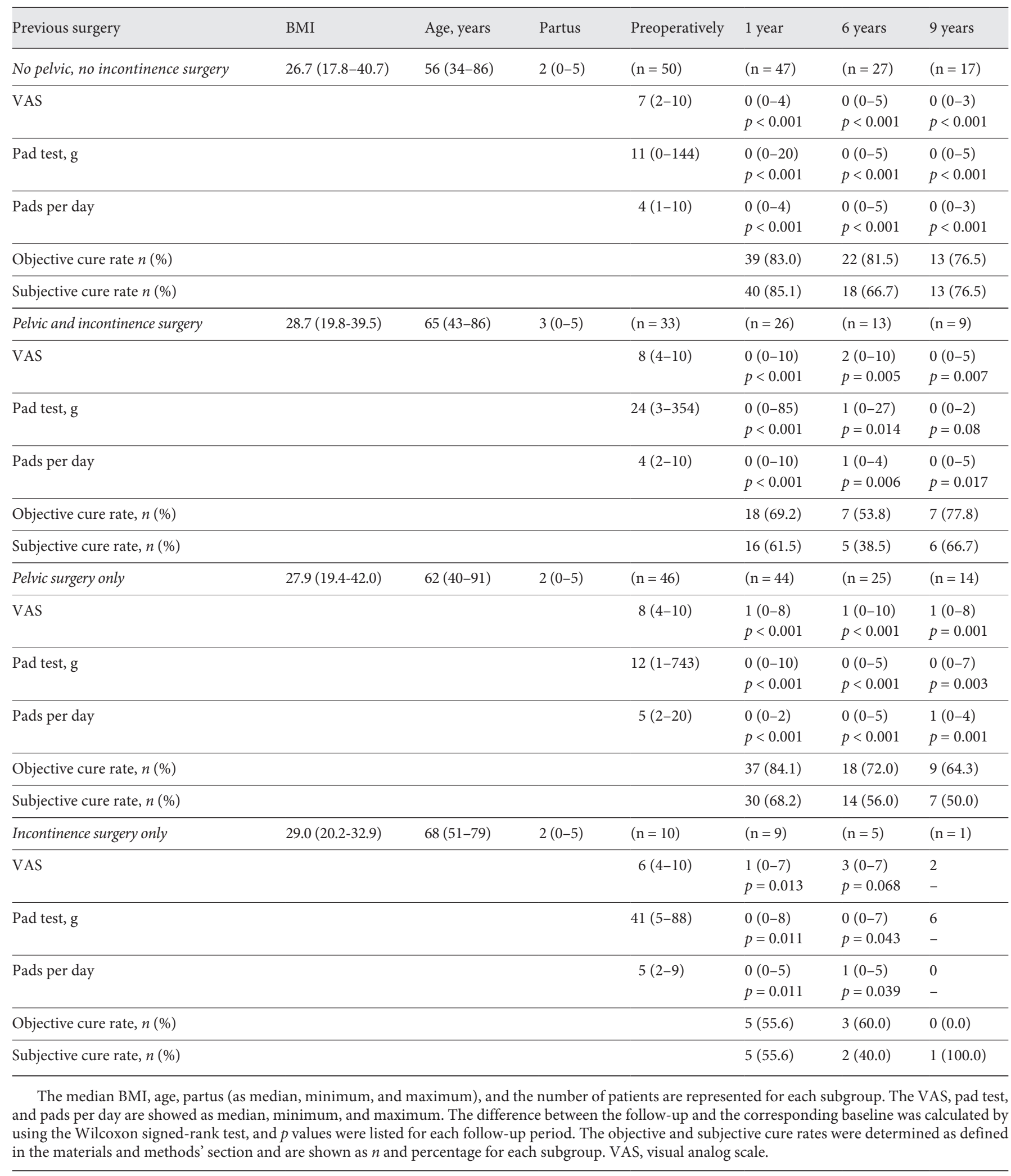


tion of the maximum flow rate. PVR is presented in Table 2 . None of the patients was feeling disturbed by the residual volume. Results regarding previous pelvic and/ or incontinence surgery are presented in Table 3. Multivariable logistic regression including the surgery groups and adjusting for age, BMI, and partus revealed no statistically significant associations with objective and subjective cure rates in all follow-up periods (online suppl. Table 2).

\section{Discussion}

The published literature stemming from original reports regarding long-term outcomes of the SPARC procedure in women with SUI is scarce [15]. An observable trend toward the litigation of mid-urethral slings could be explained by increased attention toward mesh complications over the last few years. However, mid-urethral slings have shown an acceptable risk level regarding long-term complications, with especially retropubic sling systems demonstrating an overall satisfying long-term efficacy [18]. Therefore, a further evaluation of the safety and efficacy of urethral sling systems, in particular concerning their long-term outcomes, is of great importance for the development of future treatment standards.

Regarding retropubic sling systems, TVT currently represents the method of choice with the most data (including long-term) available. Nilsson et al. [8] reported a complete cure rate of $84.7 \%$ after a median follow-up of 56 months. However, their criteria for cure were differently defined (e.g., pad test $<10 \mathrm{~g} / 24 \mathrm{~h}$ ), which makes comparisons difficult. Other work groups reported cure rates of $76.9 \%$ after 5 years and $81.3 \%$ after 7 years [ 19 , $20]$.

In our study, we were able to present comparable objective cure rates after 1,6 , and 9 years following the SPARC procedure (78.6, 71.4, and $70.7 \%$ respectively), which implicates a satisfying long-term efficacy of this surgical method. There was a slightly lower subjective cure rate after 6 and 9 years (55.7 and 65.9\%) than the 1-year follow-up evaluation (72.2\%). The notably higher incidence of urgency symptoms might represent an important factor contributing to these observations (10.0 and $9.8 \%$ compared to $1.6 \%)$. It is of paramount importance to note that the objective cure rate gives us a description of the patients' continence status at one specific moment in time, while the subjective cure rate represents patients' everyday life. Previous studies from our work group have shown similar discrepancies between objec-

Long-Term Results after SPARC Procedure for the Treatment of Female SUI tive and subjective cure rates ( 76 vs. $52 \%$ at the mean follow-up of 5.2 years) discussing that as a possible reason [15]. Another possible explanation might be our very strict definition of the subjective cure rate, which included no usage of pads during daily activities. However, a lot of women who use 1 security pad per day (in our study, nearly $50 \%$ of all women using pads) still feel continent. This phenomenon is mirrored in the higher rate of selfdescribed subjective continence at the 6- and 9-year follow-ups (67.1 and $75.6 \%$, respectively), compared to the subjective cure rates. Deval and colleagues [11] reported an objective cure rate of $90.4 \%$ and a subjective cure rate of $69.2 \%$ for a short-term mean follow-up of 11.9 months after SPARC.

In terms of complications, we equally present similar results in our recent study compared to previous, shortto mid-term results of the SPARC procedure at our department $[12,15]$. Concerning early postoperative complications, we observed postoperative urinary retention in $3 / 139(2.2 \%)$ patients. Six of $139(4.3 \%)$ patients had residual volume, whereby in these cases, a loosening of the sling was performed. Three of 139 (2.2\%) patients developed tape gliding, which was manageable by a replacement of the tape. Concerning long-term complications, we observed de novo urgency in $7 / 70(10.0 \%)$ patients after 6 years and in 4/41 (9.8\%) patients after 9 years, which correlates with previous results at our institution (10.9\%) [15]. This observation might be attributable to the increasing age of patients, since higher age and concomitant neurological and metabolic diseases represent risk factors for the development of urgency. As described previously, de novo urgency may also be related to changes in the paraurethral collagen metabolism and sclerosis around prolene tapes [21].

Recurrent lower urinary tract infections were observed in $1 / 70(1.4 \%)$ patients after 6 years and in $3 / 41(7.3 \%)$ patients after 9 years. One patient developed a symptomatic vaginal sling exposure, which made it necessary to excise the sling partially 5 years postoperatively. Another patient had an asymptomatic vaginal sling exposure without any need for further surgical treatment. Furthermore, we observed a highly significant decrease in the VAS, pad weight, number of pads, and maximum flow rate at each follow-up $(p<0.001)$.

The decrease in the flow rate represents an expected side effect after the placement of mid-urethral slings, since these exert a certain obstruction on the urethra [22]. A comparable decrease in the flow rate has been described previously by our work group $[12,15]$. Conversely, the reduction in maximal flow did not have any impact on the 
quality of life of patients in our study. We did not observe any relevant changes regarding PVR. Lower objective and subjective cure rates were observed for patients with previous surgery compared to patients without previous surgery. However, multivariable logistic regression including the surgery groups and adjusting for age, BMI, and partus revealed no statistically significant associations with objective and subjective cure rates for all follow-up periods. With increasing age and BMI, we observed a lower chance for cure (objective and subjective) after 1-year follow-up. Due to the small group sample sizes, the results should be interpreted carefully.

Our study is not devoid of limitations. One limitation is the retrospective study design, and another one is the relatively small sample size, particularly regarding the long-term follow-up evaluations. As we only included patients who personally appeared to the follow-up visits and were objectively examined, it was difficult to achieve higher patient numbers. Our results show that the SPARC procedure represents an efficient surgical method in a long-term context of up to 10 years postoperatively with an acceptable safety profile in women with SUI. The longand short-term results in our study were comparable. Moreover, high overall satisfaction rates and the reduction of the severity of bother imply a clear improvement in terms of patients' quality of life and are independent of complete dryness.

\section{Statement of Ethics}

This study was approved by the local Ethical Committee of the Medical University of Graz (no. 32-408 ex 19/20) and therefore has been performed in accordance with the ethical standards laid down in the 1964 Declaration of Helsinki and later amendments.

\section{Conflict of Interest Statement}

The authors have no conflicts of interest to declare.

\section{Funding Sources}

This study did not receive any funding.

\section{Author Contributions}

Jasarevic: protocol/project development, data collection, data analysis, and manuscript drafting/writing/editing. Jankovic: data collection and manuscript editing. Hutterer: manuscript drafting, editing, and supervision. Riedl: data analysis and manuscript editing. Pichler: data analysis and manuscript editing. Pummer: protocol/project development and supervision. Primus: protocol/ project development, data collection, and manuscript editing.

\section{References}

1 Abrams P, Cardozo L, Fall M, Griffiths D, Rosier P, Ulmsten U, et al. The standardisation of terminology of lower urinary tract function: report from the standardisation sub-committee of the international continence society. Neurourol Urodyn. 2002; 21(2):167-78.

2 Ford AARL, Cody JD, Aluko P, Ogah JA. Mid-urethral sling operations for stress urinary incontinence in women. Cochrane Database Syst Rev. 2015 Jul 1;(7):CD006375.

3 Leone Roberti Maggiore U, Bogani G, Meschia M, Sorice P, Braga A, Salvatore S, et al. Urethral bulking agents versus other surgical procedures for the treatment of female stress urinary incontinence: a systematic review and meta-analysis. Eur J Obstet Gynecol Reprod Biol. 2015 Jun; 189:48-54.

4 Giammo A, Geretto P, Ammirati E, Manassero A, Squintone L, Vercelli D, et al. Urethral bulking with Bulkamid: an analysis of efficacy, safety profile, and predictors of functional outcomes in a single-center cohort. Neurourol Urodyn. 2020 May 14.
5 Lee YS, Lee HN, Lee KS. The evolution of surgical treatment for female stress urinary incontinence: era of mid-urethral slings. Korean J Urol. 2010 Apr;51(4):223-32.

6 Fusco F, Abdel-Fattah M, Chapple CR, Creta M, La Falce S, Waltregny D, et al. Updated systematic review and meta-analysis of the comparative data on colposuspensions, pubovaginal slings, and midurethral tapes in the surgical treatment of female stress urinary incontinence. Eur Urol. 2017 Oct; 72(4):567-91.

7 Ulmsten U, Henriksson L, Johnson P, Varhos G. An ambulatory surgical procedure under local anesthesia for treatment of female urinary incontinence. Int Urogynecol J Pelvic Floor Dysfunct. 1996;7(2):81-6.

8 Nilsson CG, Kuuva N, Falconer C, Rezapour $\mathrm{M}$, Ulmsten U. Long-term results of the tension-free vaginal tape (TVT) procedure for surgical treatment of female stress urinary incontinence. Int Urogynecol J Pelvic Floor Dysfunct. 2001;12(Suppl 2):S5-8.
9 Leone Roberti Maggiore U, Finazzi Agrò E, Soligo M, Li Marzi V, Digesu A, Serati M. Long-term outcomes of TOT and TVT procedures for the treatment of female stress urinary incontinence: a systematic review and meta-analysis. Int Urogynecol J. 2017 Aug; 28(8):1119-30.

10 Dietz HP, Foote AJ, Mak HL, Wilson PD. TVT and sparc suburethral slings: a case-control series. Int Urogynecol J Pelvic Floor Dysfunct. 2004 Mar-Apr;15(2):129-31.

11 Deval B, Levardon M, Samain E, Rafii A, Cortesse A, Amarenco G, et al. A French multicenter clinical trial of SPARC for stress urinary incontinence. Eur Urol. 2003 Aug;44(2): 254-9.

12 Dalpiaz O, Primus G, Schips L. SPARC sling system for treatment of female stress urinary incontinence in the elderly. Eur Urol. 2006 Oct:50(4):826-1.

13 Primus G. One year follow-up on the SPARC sling system for the treatment of female urodynamic stress incontinence. Int J Urol. 2006 Nov;13(11):1410-4. 
14 Siddiqui K, Raj H, Flynn RJ, Grainger R, Thornhill JA. Minimally invasive treatment of female stress urinary incontinence: 100 cases using SPARC sling. Ir J Med Sci. 2008 Mar;177(1):39-42.

15 Heidler S, Ofner-Kopeinig P, Puchwein E, Pummer K, Primus G. The suprapubic arch sling procedure for treatment of stress urinary incontinence: a 5-year retrospective study. Eur Urol. 2010 May;57(5):897-901.

16 Chapple CR, Raz S, Brubaker L, Zimmern PE. Mesh sling in an era of uncertainty: lessons learned and the way forward. Eur Urol. 2013 Oct;64(4):525-9.
17 Hofner K, Hampel C, Kirschner-Hermanns R, Alloussi SH, Bauer RM, Bross S, et al. (Use of synthetic slings and mesh implants in the treatment of female stress urinary incontinence and prolapse: statement of the working group on urological functional diagnostics and female urology of the academy of the German society of urology). Urologe A. 2020 Jan; 59(1):65-71.

18 Tulokas S, Rahkola-Soisalo P, Gissler M, Mikkola TS, Mentula MJ. Long-term re-procedure rate after mid-urethral slings for stress urinary incontinence. Int Urogynecol J. 2020 Apr;31(4):727-35.

19 Tsivian A, Mogutin B, Kessler O, Korczak D, Levin S, Sidi AA. Tension-free vaginal tape procedure for the treatment of female stress urinary incontinence: long-term results. J Urol. 2004 Sep;172(3):998-1000.
20 Doo CK, Hong B, Chung BJ, Kim JY, Jung $\mathrm{HC}$, Lee KS, et al. Five-year outcomes of the tension-free vaginal tape procedure for treatment of female stress urinary incontinence. Eur Urol. 2006 Aug;50(2):333-8.

21 Falconer C, Blomgren B, Johansson O, Ulmsten U, Malmström A, Westergren-Thorsson $\mathrm{G}$, et al. Different organization of collagen fibrils in stress-incontinent women of fertile age. Acta Obstet Gynecol Scand. 1998 Jan; 77(1):87-94.

22 Tse V, Chan L. Outlet obstruction after sling surgery. BJU Int. 2011 Nov;108(Suppl 2):24- 